\title{
Two-scale Dirichlet-Neumann preconditioners for boundary refinements
}

\author{
Patrice Hauret $^{1}$ and Patrick Le Tallec ${ }^{2}$ \\ 1 Graduate Aeronautical Laboratories, MS 205-45, \\ California Institute of Technology \\ Pasadena, CA 91125, USA \\ 2 Laboratoire de Mécanique des Solides, CNRS UMR 7649, \\ Département de Mécanique, Ecole Polytechnique, \\ 91128 Palaiseau Cedex, FRANCE
}

Summary. The present work introduces simple Dirichlet-Neumann preconditioners for the resolution of elasticity problems in presence of numerous small disjoint geometric refinements on the boundary of the domain, situation which typically occurs in tire industry. Moreover, the condition number of the preconditioned system is proved to be independent of the number and the size of the small details on the boundary. Finally, as an enhancement, the second proposed preconditioner makes use of a coarse space counterbalancing the effect of essential boundary conditions on the small details, and a simple numerical academic test illustrates the increased efficiency. Further details on the motivation as well as complete proofs can be found in $[4,5]$.

\section{Introduction}

Let $\Omega \subset \mathbb{R}^{d}$ be the reference configuration of a body, partitioned into a coarse region $\Omega_{0}$ where the properties of the material are rather smooth and where a coarse approximation should be sufficient, and into small disjoint boundary regions denoted by $\left(\Omega_{k}\right)_{1 \leq k \leq K}$ where a fine discretization is required (e.g. geometrical refinements, fine behavior of the material). Such a situation typically occurs for tires, the internal structure and the surface sculptures playing the role of the coarse and fine zones, respectively. Let us denote by $\Gamma_{D}$ a part of the boundary of $\Omega$ where displacements are prescribed and by $\Gamma_{N}=\partial \Omega \backslash \Gamma_{D}$ its complementary part. Denoting by $H_{*}^{1}(\Omega):=\left\{v \in H^{1}(\Omega)^{d},\left.v\right|_{\Gamma_{D} \cap \partial \Omega}=0\right\}$ the space of admissible displacements, our model elastostatic problem consists in finding $u \in H_{*}^{1}(\Omega)$ such that:

$$
a(u, v):=\int_{\Omega} \mathbf{E}_{i j k l} \varepsilon(u)_{k l} \varepsilon(v)_{i j}=\int_{\Omega} f \cdot v+\int_{\Gamma_{N}} g \cdot v=: l(v), \quad \forall v \in H_{*}^{1}(\Omega) .
$$


Here $\mathbf{E}$ denotes the fourth order elasticity tensor, $f \in L^{2}(\Omega)^{d}$ and $g \in$ $L^{2}\left(\Gamma_{N}\right)^{d}$ the loading forces, and $\epsilon(v)=\frac{1}{2}\left(\nabla v+(\nabla v)^{t}\right)$ is the linearized strain tensor. Considering that the solution must be computed with a multi-scale approach in order to respect the characteristics of the problem, the strategy proposed in this paper consists in using:

1. mortar formulations [2, 13] on the interfaces $\Gamma_{0 k}=\partial \Omega_{0} \cap \partial \Omega_{k}$ enabling to use independent approximations in the coarse and fine regions respectively,

2. efficient Dirichlet-Neumann preconditioners [9], which we adapt so that the computational cost of the full algebraic problem remains independent (or at least weakly dependent) of the number and the size of the fine subdomains $\left(\Omega_{k}\right)_{1 \leq k \leq K}$.

The sequel is organized as follows. After the introduction of a mortar formulation (section 2), we propose two possible Dirichlet-Neumann preconditioners and state their two-scale properties (section 3). In particular, the second enhanced preconditioner makes use of a coarse space counterbalancing the effect of essential boundary conditions imposed on the boundary sculptures. A simple numerical test shows its increased efficiency for a simple academic problem. A broader perspective on the subject as well as complete proofs are given in $[4,5]$.

\section{Non-conforming formulation}

For every $0 \leq k \leq K$, let $\left(\mathcal{T}_{k ; h_{k}}\right)_{h_{k}>0}$ be a sequence of meshes of the substructure $\Omega_{k}, h_{k}$ denoting the maximal diameter of its elements. The corresponding finite-element spaces of order $q$ are denoted by $\left(V_{k ; h_{k}}\right)_{h_{k}>0} \subset H_{*}^{1}\left(\Omega_{k}\right)$. As in $[3,7]$, for stability purpose when using a discontinuous mortar formulation, interface bubbles can be added on the fine subdomains. As a consequence, we introduce the potentially enriched spaces of displacements $X_{k ; h_{k}}=V_{k ; h_{k}} \oplus B_{k ; h_{k}}$ for every $1 \leq k \leq K$ and $X_{0 ; h_{0}}=V_{0 ; h_{0}}$. For each interface $\Gamma_{0 k}, W_{k ; h_{k}}$ will stand for the trace of the local space $X_{k ; h_{k}}$ on this interface. In order to impose a weak displacement continuity between $\Omega_{0}$ and $\Omega_{k}$, a space of Lagrange multipliers $M_{k ; h_{k}}$ is introduced on the mesh $\mathcal{T}_{k ; h_{k}}$ over $\Gamma_{0 k}$. Actually, various choices of continuous or of discontinuous polynomial functions of degree $r$ can be used $[2,10,12,8,6,7]$ but in any case, they must satisfy the following fundamental assumptions:

Assumption 1 [Coercivity]. Let $u_{0} \in H^{1}\left(\Omega_{0}\right)^{d}$ and $u_{k} \in H^{1}\left(\Omega_{k}\right)^{d}$ be rigid motions, i.e. $\varepsilon\left(u_{0}\right)=0$ in $L^{2}\left(\Omega_{0}\right)^{d \times d}$ and $\varepsilon\left(u_{k}\right)=0$ in $L^{2}\left(\Omega_{k}\right)^{d \times d}$, satisfying the weak continuity requirement $\int_{\Gamma_{0 k}}\left(u_{0}-u_{k}\right) \cdot \mu=0$ for every $\mu \in M_{k ; h_{k}}$. Then $u_{0}=u_{k}$ almost everywhere on $\Gamma_{0 k}$.

Assumption 2 [Inf-sup condition]. There exists a mapping $\pi_{k}: L^{2}\left(\Gamma_{0 k}\right) \rightarrow$ $W_{k ; h_{k}}$ such that for all $v \in L^{2}\left(\Gamma_{0 k}\right)$, 


$$
\int_{\Gamma_{0 k}}\left(\pi_{k} v\right) \cdot \mu=\int_{\Gamma_{0 k}} v \cdot \mu, \quad \forall \mu \in M_{k ; h_{k}},
$$

satisfying $\left\|\pi_{k} v\right\|_{k, \frac{1}{2}} \leq C\|v\|_{k, \frac{1}{2}}$. The mesh dependent norm $\|\cdot\|_{k, \frac{1}{2}}$ introduced above is defined as in $[1,11]$ by

$$
\|v\|_{k, \frac{1}{2}}^{2}=\sum_{K \in \mathcal{T}_{k ; h_{k}}} \operatorname{diam}\left(K \cap \Gamma_{0 k}\right)^{-1} \int_{K \cap \Gamma_{0 k}} v^{2}
$$

Assumption 3 [Accuracy]. The total degree $r$ of Lagrange multipliers is bounded from below by $r \geq q-1, q$ being the total degree of the displacement shape functions.

Then, the mortar formulation of the problem of interest can be written as finding $u=\left(u_{0}, u_{1}, \ldots, u_{K}\right) \in \prod_{k=0}^{K} X_{k ; h_{k}}$ and $\lambda=\left(\lambda_{1}, \ldots, \lambda_{K}\right) \in \prod_{k=1}^{K} M_{k ; h_{k}}$ satisfying for every $v \in \prod_{k=0}^{K} X_{k ; h_{k}}$ and $\mu \in \prod_{k=1}^{K} M_{k ; h_{k}}$,

$$
\begin{aligned}
a_{0}\left(u_{0}, v_{0}\right)+\sum_{k=1}^{K} b_{0 k}\left(v_{0}, \lambda_{k}\right) & =l_{0}\left(v_{0}\right) \\
a_{k}\left(u_{k}, v_{k}\right)- & b_{k}\left(v_{k}, \lambda_{k}\right)=l_{k}\left(v_{k}\right), 1 \leq k \leq K \\
b_{0 k}\left(u_{0}, \mu_{k}\right)- & b_{k}\left(u_{k}, \mu_{k}\right)=0, \quad 1 \leq k \leq K .
\end{aligned}
$$

The above problem uses the obvious notation $a_{k}\left(u_{k}, v_{k}\right)=\int_{\Omega_{k}} \mathbf{E}_{i j m n} \varepsilon\left(u_{k}\right)_{m n} \varepsilon\left(v_{k}\right)_{i j}$,

$l_{k}\left(v_{k}\right)=\int_{\Omega_{k}} f \cdot v_{k}+\int_{\Gamma_{N} \cap \partial \Omega_{k}} g \cdot v_{k}, b_{0 k}\left(v_{0}, \mu_{k}\right)=\int_{\Gamma_{0 k}} v_{0} \cdot \mu_{k}$ and $b_{k}\left(v_{k}, \mu_{k}\right)=$ $\int_{\Gamma_{0 k}} v_{k} \cdot \mu_{k}$.

\section{Two-scale preconditioners}

In matrix notation, after elimination of the Lagrange multipliers $\lambda_{k}$ in the first equation of (1), the system becomes

$$
\left\{\begin{array}{l}
\mathbf{S}_{0} U_{0}=\overline{L_{0}}, \\
\mathbf{K}_{k}\left(\begin{array}{c}
U_{k} \\
\Lambda_{k}
\end{array}\right)=\left(\begin{array}{c}
L_{k} \\
-\mathbf{B}_{0 k} U_{0}
\end{array}\right), \quad 1 \leq k \leq K,
\end{array}\right.
$$

where $\mathbf{S}_{0}=\mathbf{A}_{0}-\sum_{k=1}^{K} \mathbf{B}_{0 k}^{t} R_{k} \mathbf{K}_{k}^{-1} R_{k}^{t} \mathbf{B}_{0 k}$ is the Schur complement matrix, and $\overline{L_{0}}=L_{0}-\sum_{k=1}^{K} \mathbf{B}_{0 k}^{t} R_{k} \mathbf{K}_{k}^{-1}\left(\begin{array}{c}L_{k} \\ 0\end{array}\right)$ the corresponding right hand side. In these definitions, the local stiffness matrix $\mathbf{K}_{k}$ and restriction operator $R_{k}$ are given by

$$
\mathbf{K}_{k}=\left(\begin{array}{cc}
\mathbf{A}_{k} & -\mathbf{B}_{k}^{t} \\
-\mathbf{B}_{k} & 0
\end{array}\right), \quad R_{k}\left(\begin{array}{c}
U_{k} \\
\Lambda_{k}
\end{array}\right)=\Lambda_{k}
$$


An iterative solver can be efficiently used to solve (2) if one is able to define a preconditioner $\tilde{\mathbf{S}}_{0}$ of the exact Schur complement $\mathbf{S}_{0}$ which is spectrally equivalent to $\mathbf{S}_{0}$, with constants independent of the number and the size of the small subdomains. When $L_{0}, . ., L_{K}$ are given, the application of such a preconditioner consists in the following operations:

1. Compute $\overline{L_{0}}$ by solving Dirichlet problems on the small subdomains prescribing zero displacements on the interfaces $\left(\Gamma_{0 k}\right)_{1 \leq k \leq K}$,

2. Solve the extended Neumann problem $\tilde{\mathbf{S}}_{0} \tilde{U}_{0}=\overline{L_{0}}$,

3. Compute $\left(\tilde{U}_{k}, \tilde{\Lambda}_{k}\right)$ over each $\Omega_{k}$ by solving the Dirichlet problem:

$$
\mathbf{K}_{k}\left(\begin{array}{c}
\tilde{U}_{k} \\
\tilde{\Lambda}_{k}
\end{array}\right)=\left(\begin{array}{c}
L_{k} \\
-\mathbf{B}_{0 k} \tilde{U}_{0}
\end{array}\right) .
$$

The most natural -and rather efficient- preconditioner consists in simply using $\tilde{\mathbf{S}}_{0}=\mathbf{A}_{0}$. This is a standard Dirichlet-Neumann preconditioner for which we prove [5]:

Proposition 1. Assuming that $\mathbf{A}_{0}$ is invertible, i.e. $\Gamma_{D} \cap \partial \Omega_{0}$ has a positive measure, the following spectral equivalence holds for all $U_{0}$ :

$$
W_{1, h}\left\langle\mathbf{S}_{0} U_{0}, U_{0}\right\rangle \leq\left\langle\mathbf{A}_{0} U_{0}, U_{0}\right\rangle \leq\left\langle\mathbf{S}_{0} U_{0}, U_{0}\right\rangle,
$$

with:

$$
\frac{1}{W_{1, h}}=1+C\left(\max _{k \in I_{1}} \frac{C_{k}}{c_{0}}+\max _{k \in I_{2}} \frac{C_{k} L_{0}}{\alpha_{0} L_{k}}\right),
$$

where $I_{1}$ (resp. $I_{2}$ ) is the set of indices $k \geq 1$ such that $\Omega_{k}$ is not fixed on its boundary (resp. is fixed on a part of its boundary), the positive constants $c_{k}$ and $C_{k}$ are such that $c_{k}|\xi|^{2} \leq \mathbf{E}_{i j m n} \xi_{m n} \xi_{i j} \leq C_{k}|\xi|^{2}$ over $\Omega_{k}$ for every symmetric matrix $\xi \in \mathbb{R}^{d \times d}, \alpha_{0}$ is the coercivity constant of the bilinear form $a_{0}$ and $L_{k}=\operatorname{diam}\left(\Omega_{k}\right)$. The constant $C>0$ is independent of the number $K$ and the size of the subdomains.

This simple choice will lack of efficiency in two simple situations:

1. a fine subdomain $\Omega_{k}(k \geq 1)$ has a small size $L_{k}<<L_{0}$ and is fixed on a part of its boundary $\left(k \in I_{2}\right)$; in this situation, because of its size, the substructure will have a rather large stiffness to interface rigid body displacements,

2. a fine subdomain $\Omega_{k}(k \geq 1)$ has several stiff modes involving interface motions (rigid links, incompressibility).

Assuming that these directions of localized interface stiffness be in very small number $N_{k}$ (this is indeed the case for interface rigid body motions), we then propose a modification of the previous preconditioner enabling to correct such a lack of efficiency. 
For all $k \geq 1$ such that $\Omega_{k}$ is fixed on a part of its boundary, we denote by $\left(e_{k}^{i}\right)_{1 \leq i \leq N_{k}}$ (with $N_{k}=6$ in general) the interface rigid motions of $\Gamma_{0 k}$ or rigid links and introduce

$$
\stackrel{\circ}{W}_{k}=\operatorname{span}\left\{e_{k}^{i}, i=1, . ., N_{k}\right\} .
$$

To each interface rigid body motion $e_{k}^{i}$, we associate its local $a_{k}$-harmonic extension $\left(u_{k}^{i}, \lambda_{k}^{i}\right) \in X_{k ; h_{k}} \times M_{k ; \delta_{k}}$ solution of

$$
\left\{\begin{array}{l}
a_{k}\left(v, u_{k}^{i}\right)-\int_{\Gamma_{0 k}} v \cdot \lambda_{k}^{i}=0, \quad \forall v \in X_{k ; h_{k}}, \\
-\int_{\Gamma_{0 k}} u_{k}^{i} \cdot \mu=-\int_{\Gamma_{0 k}} e_{k}^{i} \cdot \mu, \quad \forall \mu \in M_{k ; \delta_{k}} .
\end{array}\right.
$$

These solutions span two small local spaces

$$
\begin{aligned}
& \stackrel{\circ}{X}_{k}=\operatorname{span}\left\{u_{k}^{i}, i=1, . ., N_{k}\right\} \subset X_{k ; h_{k}}, \\
& \stackrel{\circ}{M}_{k}=\operatorname{span}\left\{\lambda_{k}^{i}, i=1, . ., N_{k}\right\} \subset M_{k ; \delta_{k}} .
\end{aligned}
$$

If $k \geq 1$ is such that $\Omega_{k}$ is not fixed on its boundary, we adopt

$$
\stackrel{\circ}{W}_{k}=\stackrel{\circ}{M}_{k}=\{0\} .
$$

Then, instead of finding $U_{0}$ such that $\mathbf{S}_{0} U_{0}=\overline{L_{0}}$, we propose to compute $u_{0} \in X_{0 ; h_{0}},\left(u_{k}\right) \in\left(\AA^{\prime}\right)_{1 \leq k \leq K},\left(\lambda_{k}\right) \in\left(\stackrel{\circ}{M}_{k}\right)_{1 \leq k \leq K}$ solution of the coupled problem

$$
\left\{\begin{array}{l}
a_{0}\left(u_{0}, v_{0}\right)+\sum_{k=1}^{K} \int_{\Gamma_{0 k}} v_{0} \cdot \lambda_{k}=\overline{l_{0}}\left(v_{0}\right), \quad \forall v_{0} \in X_{0 ; h_{0}}, \\
a_{k}\left(u_{k}, v_{k}\right)-\int_{\Gamma_{0 k}} v_{k} \cdot \lambda_{k}=0, \quad \forall v_{k} \in \stackrel{\circ}{X}_{k}, \quad 1 \leq k \leq K, \\
-\int_{\Gamma_{0 k}} u_{k} \cdot \mu_{k}=-\int_{\Gamma_{0 k}} u_{0} \cdot \mu_{k}, \quad \forall \mu_{k} \in \stackrel{\circ}{M}_{k}, \quad 1 \leq k \leq K .
\end{array}\right.
$$

This amounts to reduce the local substructure response to the harmonic extension of its stiff interface modes, which belongs to $\dot{X}_{k}$. We introduce the matrix $\mathbf{I}_{0 k}=\boldsymbol{\Lambda}_{k}^{t} \mathbf{B}_{0 k}$ where $\boldsymbol{\Lambda}_{k}^{t}=\left[\Lambda_{k}^{1}, . ., \Lambda_{k}^{N_{k}}\right]^{t}$ is the matrix built with the multipliers computed in (3), and the restriction $\mathbf{\AA}_{k}$ of the displacement stiffness matrix $\mathbf{A}_{k}$ to the local space $\dot{X}_{k}$

$$
\left(\stackrel{\AA}{A}_{k}\right)_{i j}=\left(U_{k}^{i}\right)^{t} \mathbf{A}_{k} U_{k}^{j}=a_{k}\left(u_{k}^{j}, u_{k}^{i}\right)=\int_{\Gamma_{0 k}} u_{k}^{j} \cdot \lambda_{k}^{i},
$$

where (3) has been used. Exploiting (5) to reformulate (4)-2,(4)-3, the system (4) can be rewritten after some algebraic elimination as

$$
\tilde{\mathbf{S}}_{0} U_{0}=\overline{L_{0}}
$$


with a new approximate Schur complement given by

$$
\begin{aligned}
\tilde{\mathbf{S}}_{0} & =\mathbf{A}_{0}+\sum_{k=1}^{K} \mathbf{I}_{0 k}^{t} \stackrel{\circ}{k}_{k}^{-t} \mathbf{I}_{0 k} \\
& =\mathbf{A}_{0}+\sum_{k=1}^{K} \mathbf{B}_{0 k}^{t} \boldsymbol{\Lambda}_{k} \stackrel{\AA}{\mathbf{A}}_{k}^{-t} \boldsymbol{\Lambda}_{k}^{t} \mathbf{B}_{0 k} .
\end{aligned}
$$

The complexity of its inversion is much smaller than solving $\mathbf{S}_{0} U_{0}=\overline{L_{0}}$ because each local problem (3) used in the construction of $\tilde{\mathbf{S}}_{0}$ only involves a subspace of displacements of dimension $N_{k}$. Moreover, we prove in [5] that:

Proposition 2. For all $U_{0}$, the following spectral equivalence holds

$$
W_{1, h}\left\langle\mathbf{S}_{0} U_{0}, U_{0}\right\rangle \leq\left\langle\tilde{\mathbf{S}}_{0} U_{0}, U_{0}\right\rangle \leq\left\langle\mathbf{S}_{0} U_{0}, U_{0}\right\rangle
$$

with

$$
\frac{1}{W_{1, h}}=C\left(1+\max _{1 \leq k \leq K} \frac{C_{k}}{c_{0}}\right) .
$$

The constant $C>0$ is independent of the number $K$ and the size of the subdomains.

\section{Numerical illustration}

Let us consider here a two-scale beam (as represented on figure 1) whose both tips are clamped. The material is elastic, isotropic, homogeneous in each substructure, and the displacements under loading are computed by a preconditioned conjugate gradient method. Figure 2 illustrates the advantage of the enhanced Dirichlet-Neumann preconditioner when two small substructures are clamped. In conformity with the announced results, the gain in efficiency is independent of the ratio of Young moduli between the fine and coarse zones. Moreover, a factor 3 improvement is achieved in the number of iterations, and roughly speaking in the time of computation. Finally, it is shown in [5] that such a preconditioner can be used as an efficient quasi-tangent operator in the nonlinear framework as soon as boundary geometrical details are sufficiently soft.

\section{Conclusion}

The domain-decomposition based preconditioners proposed here achieve scaleindependent performances. They should be extended to cases where the details overlap the coarse region in the line of the fictitious domains approach, and also to cases where the details are not disjoint but constitute a continuous belt along the boundary. 


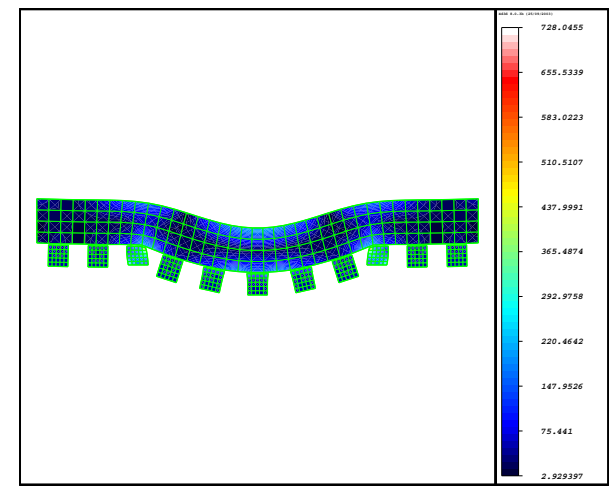

Fig. 1. Maximal stress distribution on a deformed configuration of our two-scale model problem where two of the details are clamped on their lower face.
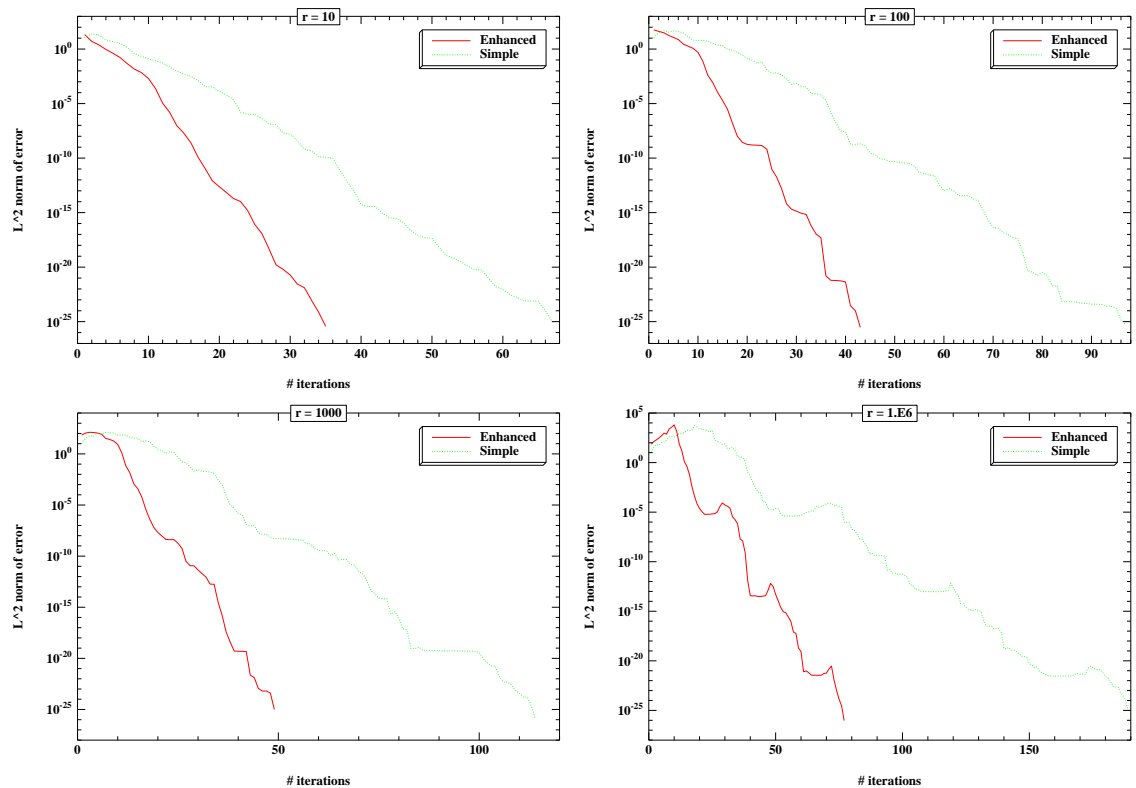

Fig. 2. Convergence of the simple and enhanced Dirichlet-Neumann algorithms for different values of the ratio $r$ of Young moduli between the fine and coarse subdomains.

Acknowledgement. The authors gratefully acknowledge the financial support of Michelin Tire Company during the completion of this work at the Center of Applied Mathematics (CMAP), Ecole Polytechnique, and Professor François Jouve for providing his finite element code in which the presented ideas have been implemented. 


\section{References}

1. A. Agouzal and J.M. Thomas. Une méthode d'éléments finis hybrides en décomposition de domaines. RAIRO M2AN, 29:749-764, 1995.

2. C. Bernardi, Y. Maday, and A.T. Patera. Domain decomposition by the mortar element method. In H.G. Kaper ans M. Garbey, editor, Asymptotic and Numerical Methods for Partial Differential Equations with Critical Parameters, pages 269-286. N.A.T.O. ASI, Kluwer Academic Publishers, 1993.

3. F. Brezzi and D. Marini. Error estimates for the three-field formulation with bubble stabilization. Math. Comp., 70:911-934, 2000.

4. P. Hauret. Méthodes numériques pour la dynamique des structures non-linéaires incompressibles à deux échelles (Numerical methods for the dynamic analysis of two-scale incompressible nonlinear structures). PhD thesis, Ecole Polytechnique, 2004.

5. P. Hauret and P. Le Tallec. Dirichlet-neumann preconditioners for elliptic problems with small disjoint geometric refinements on the boundary. Technical Report 552, CMAP - Ecole Polytechnique, september 2004.

6. P. Hauret and P. Le Tallec. A stabilized discontinuous mortar formulation for elastostatics and elastodynamics problems, part i: abstract framework. Technical Report 553, CMAP - Ecole Polytechnique, september 2004.

7. P. Hauret and P. Le Tallec. A stabilized discontinuous mortar formulation for elastostatics and elastodynamics problems, part ii: discontinuous Lagrange multipliers. Technical Report 554, CMAP - Ecole Polytechnique, september 2004.

8. C. Kim, R.D. Lazarov, J.E. Pasciak, and P.S. Vassilevski. Multiplier spaces for the mortar finite element method in three dimensions. SIAM J. Numer. Anal., 39:519-538, 2001.

9. A. Quarteroni and A. Valli. Domain Decomposition Methods for Partial Differential Equations. Oxford University Press, 1999.

10. P. Seshaiyer. Non-conforming hp finite element methods. PhD thesis, University of Maryland, 1998.

11. B.I. Wohlmuth. Hierarchical a posteriori error estimators for mortar finite element methods with Lagrange multipliers. SIAM J. Numer. Anal., 36:1636-1658, 1999.

12. B.I. Wohlmuth. A mortar finite element method using dual spaces for the Lagrange multiplier. SIAM J. Numer. Anal., 38:989-1012, 2000.

13. B.I. Wohlmuth. Discretization methods and iterative solvers based on domain decomposition. Springer, 2001. 